\title{
Recurrent Inversion Events at 17q21.31 Microdeletion Locus Are Linked to the MAPT H2 Haplotype
}

\author{
P.N. Rao ${ }^{a}$ W. Li ${ }^{b} \quad$ L.E.L.M. Vissers ${ }^{c} \quad$ J.A. Veltman ${ }^{c}$ R.A. Ophoff ${ }^{b, d}$ \\ ${ }^{a}$ Department of Pathology and Laboratory Medicine and ${ }^{\mathrm{b} C}$ Center for Neurobehavioral Genetics, Neuropsychiatric \\ Institute, University of California Los Angeles, Los Angeles, Calif., USA; 'Department of Human Genetics, \\ Nijmegen Centre for Molecular Life Sciences, Radboud University Nijmegen Medical Centre, Nijmegen, and \\ ${ }^{d}$ Department of Medical Genetics and Rudolf Magnus Institute of Neuroscience, University Medical Center Utrecht, \\ Utrecht, The Netherlands
}

\section{Key Words}

17q21.31 microdeletion - Genomic disorder $\cdot \mathrm{H} 1$ and $\mathrm{H} 2$ haplotypes $\cdot$ Inversion polymorphism $\cdot$ Recurrent

\begin{abstract}
The chromosomal band 17q21.31, containing the microtubule-associated protein tau (MAPT) gene, is a hotspot for chromosomal rearrangements. It is known to contain a common inversion polymorphism of approximately $900 \mathrm{~kb}$ in populations with European ancestry. The inverted configuration is linked to a distinct MAPT haplotype, $\mathrm{H} 2$, which is relatively common in Europeans but nearly absent in Asian and African populations. Recent studies have demonstrated that the $\mathrm{H} 2$ haplotype is ancestral in hominoids, and under positive selection in Europeans. This haplotype is also linked to events leading to the 17q21.31 microdeletion syndrome, one of the most common causes of 'idiopathic' mental retardation in people of European descent. We performed direct analysis of the chromosome structure by fluorescence in situ hybridization and observed heterozygosity of the inversion status for the $\mathrm{H} 2$ chromosomes, but not for the $\mathrm{H} 1$ haplotype. Inversion heterozygosity was also observed in a mother homozygous for the $\mathrm{H} 2$ haplotype, who transmitted the
\end{abstract}

chromosome with the deletion to a proband with 17q21.31 microdeletion syndrome. Our results highlight an allele-specific sensitivity to chromosome rearrangements and suggest that it is the heterozygosity of inversion status that predisposes to the 17q21.31 microdeletion syndrome.

Copyright $\odot 2010$ S. Karger AG, Basel

It has been postulated that genomic low copy repeats (LCRs) predispose to non-allelic homologous recombination or uneven crossing-over events leading to duplications, deletions and inversions. Changes in gene dosage or rearrangements in chromosomal architecture structure mediated by LCRs can lead to several human genomic disorders [Lupski, 2007]. These loci have also been reported to contain genomic inversion polymorphisms, in addition to being hotspots for recurrent genomic deletions. Heterozygous carriers of chromosomal inversion polymorphisms are thought to be more likely to produce offspring with genomic deletion syndromes [Osborne et al., 2001; Gimelli et al., 2003]. High-resolution genome analysis in patients with unexplained mental retardation has led to the identification of the chromosome 17q21.31 microdeletion syndrome [Koolen et al., 2006; Sharp et al., 2006;

\section{KARGER}

Fax +41613061234

E-Mail karger@karger.ch

www.karger.com (c) 2010 S. Karger AG, Basel

Accessible online at: www.karger.com/cgr
Roel A. Ophoff

Center for Neurobehavioral Genetics, Neuropsychiatric Institute

University of California Los Angeles

Los Angeles, CA 90095 (USA)

Tel. +1 310794 9602, Fax +1 310794 9613, E-Mail Ophoff@ucla.edu 
Shaw-Smith et al., 2006]. The deleted region coincides with a known inversion polymorphism of $\sim 900 \mathrm{~kb}$ containing the microtubule-associated protein tau (MAPT) gene and it is flanked by LCRs [Stefansson et al., 2005; Zody et al., 2008]. It is estimated that this microdeletion is one of the most common causes for idiopathic mental retardation in subjects of European descent, although no such cases have been reported from non-European populations [Koolen et al., 2006]. Two highly divergent MAPT haplotypes, $\mathrm{H} 1$ and $\mathrm{H} 2$, were described, with distinct linkage disequilibrium patterns across the region. Reconstruction of the clones used in the human genome assembly revealed that the $\mathrm{H} 2$ haplotype is inverted and that there are some other differences in genomic structure between the $\mathrm{H} 1$ and $\mathrm{H} 2$ haplotypes. $\mathrm{H} 2$ haplotypes are relatively common in Europeans, occurring in $20 \%$ of the population, whereas they are rare to almost absent in African and Asian populations. In the Icelandic population it was observed that $\mathrm{H} 2$ is associated with increased fecundity and higher recombination rates in female carriers [Stefansson et al., 2005]. It has been suggested that Homo neanderthalensis contributed the ancestral $\mathrm{H} 2$ haplotype to Homo sapiens and that the $\mathrm{H} 2$ haplotype has been under selection pressure ever since [Hardy et al., 2005]. The $\mathrm{H} 1$ and $\mathrm{H} 2$ structures represent different clades of haplotypes without any evidence of inter-recombination events, probably due to the inverted status of $\mathrm{H} 2$. The $\mathrm{H} 1$ configuration is associated with Alzheimer's disease [Myers et al., 2005], progressive supranuclear palsy [Conrad et al., 1997], and Parkinson disease [Zabetian et al., 2007], while the $\mathrm{H} 2$ haplotype is linked to the recurrent deletion events associated with the $17 \mathrm{q} 21.31$ microdeletion syndrome. The intriguing genomic structure, history of selection, and involvement in disease led us to investigate the $17 q 21.31$ inversion polymorphism in more detail. The identification of the $\mathrm{H} 2$ inversion in the literature was primarily based on reconstruction of haplotype-specific clone contig mapping, but without more informative, direct methods of chromosome analysis such as fluorescence in situ hybridization (FISH). One study reported FISH results on mechanically stretched chromosomes in a subject homozygous for $\mathrm{H} 1$ and $\mathrm{H} 2$, respectively, confirming the inverted configuration of the $\mathrm{H} 2$ haplotype [Gijselinck et al., 2006]. We hypothesized that common inversion polymorphisms flanked by large, almost identical LCRs in opposite orientation, are likely to undergo recurrent inversion events [Mehan et al., 2004]. If this is true for the 17q21.31 locus, one would expect to find both inversion configurations on chromosomes with the $\mathrm{H} 1$ and $\mathrm{H} 2$ haplotypes in the population.

\section{Materials and Methods}

We identified lymphoblast cell lines (LCLs) of HapMap subjects who were homozygous for either the $\mathrm{H} 1$ or $\mathrm{H} 2$ haplotypes using the available SNP data of rs1800547 and rs9468, as described previously [Stefansson et al., 2005]. In brief, rs1800547 is located downstream of MAPT exon 4 and rs9468 is located in exon 9 of the same gene. $\mathrm{H} 2$ is characterized by the alleles $\mathrm{G}$ and C of SNPs rs1800547 and rs9468, respectively. Additional homozygous carriers of $\mathrm{H} 1$ and $\mathrm{H} 2$ were identified in the Human Variation Collection available through the Coriell Institute for Medical Research, New Jersey, USA. Lymphoblast cell lines of these subjects were obtained from the Coriell Cell repository, and the SNP genotyping was performed using TaqMan real-time PCR (Applied Biosystems, Foster City, Calif., USA). Lymphoblast cell lines of probands with the $17 \mathrm{q} 21$ microdeletion and their unaffected parents were generated using standard methods at the University Medical Center Nijmegen, the Netherlands. These anonymized frozen samples were shipped to UCLA for FISH analysis and $\mathrm{H} 1 / \mathrm{H} 2$ genotyping.

For the FISH analysis, the 23 LCLs were harvested after a 24-h media-starvation to synchronize the cells to being mostly in the G1 phase. Standard FISH methods were employed. Dual-color FISH was done using 3 BAC probes (RP11-403G3, RP11-256F16, RP11-80L9) (fig. 1) directly labeled by nick-translation with biotin and digoxigenin and hybridized overnight to interphase cells (probes are less than $1 \mathrm{Mb}$ apart) at $37^{\circ} \mathrm{C}$. The cells were stained with DAPI, and a minimum of 50 interphase cells were analyzed under a Nikon fluorescence microscope equipped with appropriate filters. The images were captured with Isis Imaging System (Metasystems Group Inc., Waltham, Mass., USA). The orientation of the dual-colored signals was scored independently by 2 persons. A green-red-green (GRG) signal pattern is the $\mathrm{H} 1$ or direct orientation, while a GGR signal pattern on one or 2 chromosomal homologs represents the inverted orientation (figs. 1, 2).

We performed 3-locus interphase FISH experiments and examined the chromosome structure of $17 \mathrm{q} 21.31$ in a targeted study sample of subjects homozygous for the $\mathrm{H} 1$ and $\mathrm{H} 2$ haplotypes, and also of 3 patients with 17q21.31 microdeletion syndrome and their unaffected parents who have been previously described [Koolen et al., 2006].

FISH analyses were also performed on 7 cell lines derived from individuals of European descent that were homozygous for the $\mathrm{H} 2$ allele (based on the diagnostic SNPs rs1800547 and rs9468). Another 7 cell lines were examined from HapMap subjects, also of European descent and who were either homozygous for H1 ( $\mathrm{n}=$ $5)$ or heterozygous $\mathrm{H} 1 / \mathrm{H} 2(\mathrm{n}=2)$. In a third series of FISH analyses, we established the inversion status of $317 \mathrm{q} 21$ deletion patients and their parents.

\section{Results and Discussion}

Results of FISH analysis are listed in table 1 and online supplementary table 1 (for online suppl. material, see www.karger.com/doi/10.1159/000315901). In summary, we identified 8 homozygous $\mathrm{H} 1$ carriers, who were all found to be homozygous for the non-inverted 'wild-type' 


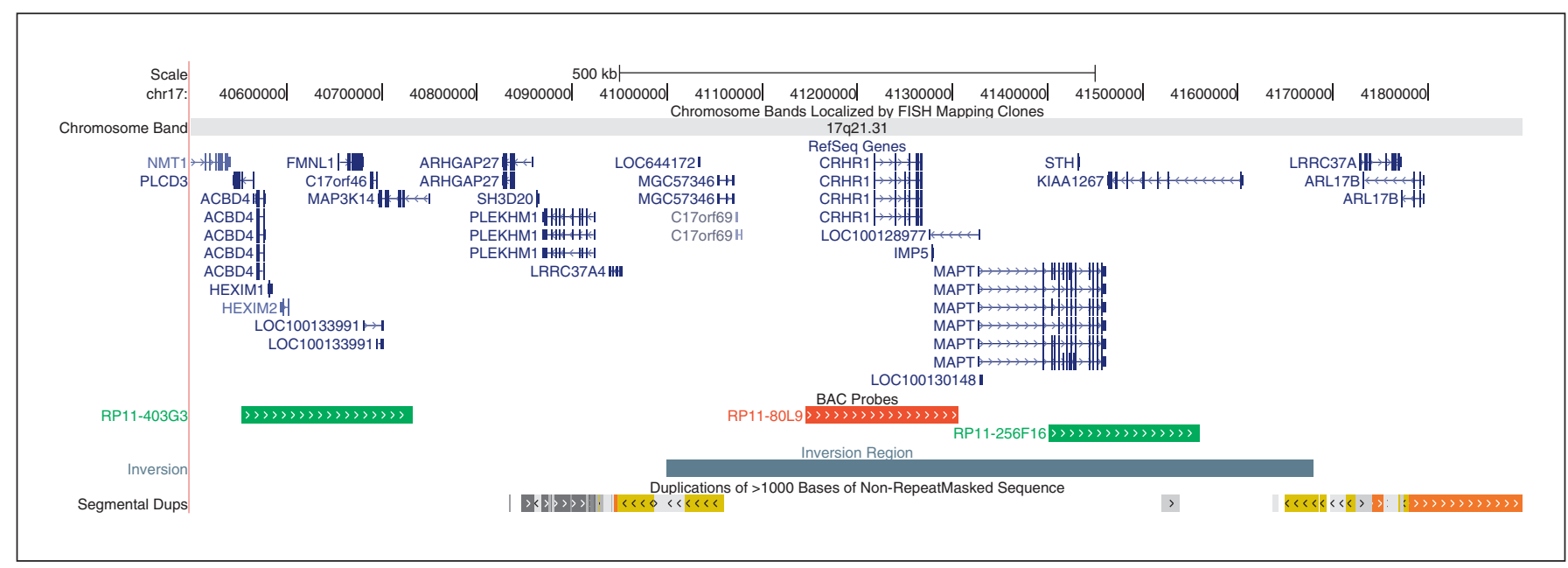

Fig. 1. Schematic overview of the $17 \mathrm{q} 21.31$ region in direct orientation corresponding with the H1 haplotype, location of segmental duplication regions, and the location of BAC probes used for FISH analysis.

Fig. 2. Representative interphase cells with the FISH probes showing the orientation (green-red-green: non-inverted orientation and red-green-green: inverted orientation) of the 3 BAC probes from one of the nuclear families. A 31919-Father $(\mathrm{H} 1 / \mathrm{H} 1)$, B 31918-Mother (H2/H2), C 31920-Proband with $17 \mathrm{q} 21$ deletion (H1/-).


Table 1. Overview of inversion status by $\mathrm{H} 1$ and $\mathrm{H} 2$ haplotypes reveals significantly more recurrent inversion events in the $\mathrm{H} 2$ lineage

\begin{tabular}{|c|c|c|c|c|}
\hline \multirow{2}{*}{$\begin{array}{l}\text { Genotype } \\
\mathrm{H} 1 / \mathrm{H} 2 \\
\text { haplotypes }\end{array}$} & \multicolumn{3}{|l|}{ FISH status } & \multirow[t]{2}{*}{ Total } \\
\hline & $\begin{array}{l}\text { Homozygous } \\
\text { 'wildtype' }\end{array}$ & $\begin{array}{l}\text { Heterozygous } \\
\text { inversion }\end{array}$ & $\begin{array}{l}\text { Homozygous } \\
\text { inversion }\end{array}$ & \\
\hline $\mathrm{H} 1 / \mathrm{H} 1$ & 8 & 0 & 0 & 8 \\
\hline $\mathrm{H} 1 / \mathrm{H} 2$ & 0 & 4 & 0 & 4 \\
\hline $\mathrm{H} 2 / \mathrm{H} 2$ & 0 & 5 & 3 & 8 \\
\hline
\end{tabular}

configuration and FISH analysis of the 4 heterozygous $\mathrm{H} 1 / \mathrm{H} 2$ carriers revealed a heterozygous inversion state of the 17q21 locus (table 1 and online suppl. table 1). However, FISH analysis of the 8 homozygous $\mathrm{H} 2$ carriers showed that 5 subjects were heterozygous for inversion status, while 3 were homozygous for the inversion. Inversion heterozygosity of all $\mathrm{H} 1 / \mathrm{H} 2$ subjects supports the link of $\mathrm{H} 2$ with inversion and of $\mathrm{H} 1$ with direct orientation. Of the $16 \mathrm{H} 1$ chromosomes from the homozygote carriers, none were inverted (0\%), while in the $16 \mathrm{H} 2$ chromosomes from the homozygote carriers we observed 5 non-inverted configurations (31\%). The over-representation of evident inversion events on the $\mathrm{H} 2$ haplotype is significantly higher than those observed on the $\mathrm{H} 1$ haplotype ( $\mathrm{p}=0.043$; Fisher's exact test).

Based on our results we conclude that recurrent inversion events of the 17q21.31 inversion region are preferentially associated with the $\mathrm{H} 2$ haplotype. For all three 
17q21.31 microdeletion patients included in our study, it had been reported that the de novo deletion occurred on the $\mathrm{H} 2$ haplotype carried by one of the parents [Koolen et al., 2008]. In our screen of these probands, 2 of the 3 parents transmitting the disease-related chromosome were heterozygous $\mathrm{H} 1 / \mathrm{H} 2$, while one was homozygous for the $\mathrm{H} 2$ haplotype. Our FISH observation on the heterozygosity of inversion status for this homozygous $\mathrm{H} 2$ carrier confirms the 'fragile' nature of $\mathrm{H} 2$, but also suggests that it is the heterozygosity of inversion status which predisposes to such de novo deletion events (fig. 2). A recent study on 22 patients showed that while the parent-of-origin of the deleted chromosome 17 always carries at least one $\mathrm{H} 2$ allele, in 6 of the 20 cases for which transmission could be established, the parent-of-origin was in fact homozygous for the $\mathrm{H} 2$ allele [Koolen et al., 2008]. If inversion status is the mediating factor for such deletion events, all the parents in this study, including the 6 homozygous $\mathrm{H} 2$ carriers, must be heterozygous for the inverted orientation. This implies that $23 \%$ of $\mathrm{H} 2$ chromosomes in these parents are in the non-inverted orientation, which is well within the range of $31 \%$ observed in our own study.

The strong linkage disequilibrium pattern separating the $\mathrm{H} 1$ and $\mathrm{H} 2$ lineages reveals a lack of recombination between the 2 haplotypes, probably due to the opposite orientations of $\mathrm{H} 1$ and $\mathrm{H} 2$. However, our findings suggest that up to one third of $\mathrm{H} 2$ chromosomes carry the noninverted configuration, which should recombine with $\mathrm{H} 1$ and result in mixed haplotypes in European populations. Yet the unusual pattern of haplotype diversity seen between $\mathrm{H} 1$ and $\mathrm{H} 2$ suggests the absence of such events in recent human history in Europe. The lack of intermingling of haplotypes implies that there are other structural differences that prohibit recombination events between the 2 haplotypes. These differences may be located within the inversion region or in the immediate flanking LCR regions. A BAC-based reconstruction of this region has shown that genomic differences between the haplotypes do indeed exist [Cruts et al., 2005; Stefansson et al., 2005; Zody et al., 2008], thereby possibly explaining this phenomenon. A comparative and detailed sequence analysis of the 17q21.31 inversion region recently favored the $\mathrm{H} 2$ configuration as the likely ancestral state and established that inversions have occurred in humans and chimpanzees independently [Zody et al., 2008]. This study further showed extensive $\mathrm{H} 2$-specific sequence homogenization of the duplicated sequences in this region, resulting in 3 times as many duplicated base pairs in $\mathrm{H} 2$ than $\mathrm{H} 1$, which could explain the 'fragile' nature of the $\mathrm{H} 2$ lineage leading to inversion and deletion events. Comparison of $\mathrm{H} 2$ haplotypes in both chromosome configurations is needed to establish whether these inverted $\mathrm{H} 2$ chromosomes are derived from single events in early human history or whether they represent more recent occurrences in European populations only. Our observation of both chromosome configurations in non-human primates with the ancestral $\mathrm{H} 2$ lineage highlights the possibility of ongoing recurrent inversion events in the genomic history of $H$. sapiens. The evidence that the relatively unstable $\mathrm{H} 2$ haplotype is involved in positive selection makes the 17q21.31 region even more appealing for further studies.

\section{Acknowledgements}

We gratefully acknowledge Drs. David Koolen and Bert de Vries for collecting cell lines and the clinical evaluation of patients with $17 \mathrm{q}$ microdeletions and their parents. This work was supported by grants from The Netherlands Organization for Health Research and Development (L.E.L.M.V. and J.A.V.), and the European AnEUploidy project (J.A.V.). The National Institutes of Health supported this study via grant GM068875 to R.A.O. The authors thank Jackie Senior for proofreading the manuscript.

\section{References}

Conrad C, Andreadis A, Trojanowski JQ, Dickson DW, Kang D, et al: Genetic evidence for the involvement of tau in progressive supranuclear palsy. Ann Neurol 41:277-281 (1997).

Cruts M, Rademakers R, Gijselinck I, van der Zee J, Dermaut B, et al: Genomic architecture of human 17q21 linked to frontotemporal dementia uncovers a highly homologous family of low-copy repeats in the tau region. Hum Mol Genet 14:1753-1762 (2005).
Gijselinck I, Bogaerts V, Rademakers R, van der Zee J, Van Broeckhoven C, Cruts M: Visualization of MAPT inversion on stretched chromosomes of tau-negative frontotemporal dementia patients. Hum Mutat 27:10571059 (2006)

Gimelli G, Pujana MA, Patricelli MG, Russo S, Giardino D, et al: Genomic inversions of human chromosome 15q11-q13 in mothers of Angelman syndrome patients with class II (BP2/3) deletions. Hum Mol Genet 12:849858 (2003).
Hardy J, Pittman A, Myers A, Gwinn-Hardy K, Fung HC, et al: Evidence suggesting that Homo neanderthalensis contributed the $\mathrm{H} 2$ MAPT haplotype to Homo sapiens. Biochem Soc Trans 33:582-585 (2005).

Koolen DA, Vissers LE, Pfundt R, de Leeuw N, Knight SJ, et al: A new chromosome 17q21.31 microdeletion syndrome associated with a common inversion polymorphism. Nat Genet 38:999-1001 (2006). 
Koolen DA, Sharp AJ, Hurst JA, Firth HV, Knight SJ, et al: Clinical and molecular delineation of the $17 \mathrm{q} 21.31$ microdeletion syndrome. J Med Genet 45:710-720 (2008).

Lupski JR: Genomic rearrangements and sporadic disease. Nat Genet 39:S43-S47 (2007).

Mehan MR, Freimer NB, Ophoff RA: A genomewide survey of segmental duplications that mediate common human genetic variation of chromosomal architecture. Hum Genomics 1:335-344 (2004).

Myers AJ, Kaleem M, Marlowe L, Pittman AM, Lees AJ, et al: The H1c haplotype at the MAPT locus is associated with Alzheimer's disease. Hum Mol Genet 14:2399-2404 (2005).
Osborne LR, Li M, Pober B, Chitayat D, Bodurtha J, et al: A 1.5 million-base pair inversion polymorphism in families with Williams-Beuren syndrome. Nat Genet 29: 321-325 (2001).

Sharp AJ, Hansen S, Selzer RR, Cheng Z, Regan $\mathrm{R}$, et al: Discovery of previously unidentified genomic disorders from the duplication architecture of the human genome. Nat Genet 38:1038-1042 (2006).

-Shaw-Smith C, Pittman AM, Willatt L, Martin $\mathrm{H}$, Rickman L, et al: Microdeletion encompassing MAPT at chromosome $17 \mathrm{q} 21.3$ is associated with developmental delay and learning disability. Nat Genet 38:1032-1037 (2006).
Stefansson H, Helgason A, Thorleifsson G, Steinthorsdottir V, Masson G, et al: A common inversion under selection in Europeans. Nat Genet 37:129-137 (2005).

-Zabetian CP, Hutter CM, Factor SA, Nutt JG, Higgins DS, et al: Association analysis of MAPT H1 haplotype and subhaplotypes in Parkinson's disease. Ann Neurol 62:137-144 (2007).

Zody MC, Jiang Z, Fung HC, Antonacci F, Hillier LW, et al: Evolutionary toggling of the MAPT 17q21.31 inversion region. Nat Genet 40:1076-1083 (2008) 